\title{
Erratum to: Lê cycles and Milnor classes
}

\author{
R. Callejas-Bedregal • M. F. Z. Morgado • \\ J. Seade
}

Published online: 1 July 2014

(C) Springer-Verlag Berlin Heidelberg 2014

\section{Erratum to: Invent Math DOI 10.1007/s00222-013-0450-7}

In the original article we introduced a concept of global Lê cycles of singular hypersurfaces in compact complex manifolds, and we gave a formula (Theorem 1) relating these with the Milnor classes of the corresponding hypersurface. There are two errors in the original article that we discuss and correct below. The first of these is in the definition we gave of the global Lê cycles (Definition 3.2). The second comes from the fact that the ambient smooth complex space $M$ should be contained in a projective space in order to use Piene's

The online version of the original article can be found under doi:10.1007/s00222-013-0450-7.

R. Callejas-Bedregal

Centro de Ciências Exatas e da Natureza-Campus I, Universidade Federal

da Paraíba-UFPb, Cidade Universitária s/n Castelo Branco, João Pessoa, PB, Brazil

e-mail: roberto@mat.ufpb.br

M. F. Z. Morgado

Instituto de Biociências Letras e Ciências Exatas, Universidade Estadual Paulista-UNESP,

Rua Cristóvão Colombo, 2265, Jd. Nazareth, S.J. do Rio Preto, SP, Brazil

e-mail: mfzmorgado@hotmail.com

J. Seade $(\varangle)$

Instituto de Matemáticas, Unidad Cuernavaca, Universidad Nacional Autónoma de

México-UNAM, Universidad s/n, Lomas de Chamilpa, C.P. 62210 Cuernavaca,

Morelos, Mexico

e-mail: jseade@im.unam.mx 
formula in [5, Théorème 3] for the Mather classes. Hence the very ampleness hypothesis on the line bundle $L$ is no longer needed.

The correct statement of Theorem 1 is:

Theorem 1.1 Let $M$ be a smooth complex submanifold of $\mathbb{P}^{N}$ of dimension $n+1$, let $Z$ be the hypersurface in $M$ defined by the set of zeroes of a reduced holomorphic section s of a line bundle $L$ on M. Set $h:=c_{1}\left(\left.\mathcal{O}_{\mathbb{P}^{N}}(1)\right|_{Z}\right)$ and denote by $\mathcal{M}_{k}(Z)$ the $k$-th Milnor class of $Z$ as defined in Definition 5.1. Then, for each $k=0, \ldots, r=\operatorname{dim}\left(Z_{\text {sing }}\right)$, there are cycles, obtained with respect to the choice of a linear subspace of $\mathbb{P}^{N}$, which give rise to well defined classes $\Lambda_{k}(Z)$ of $Z$ in the Chow group and integral homology group of $Z$, that we call the global Lê classes of $Z$, and these are related to the Milnor classes $\mathcal{M}_{k}(Z)$ by the formulas:

$$
\mathcal{M}_{k}(Z)=\sum_{j \geq 0} \sum_{i \geq k+j}(-1)^{i+j}\left(\begin{array}{c}
i+1 \\
k+j+1
\end{array}\right) c_{1}\left(\left.L\right|_{Z}\right)^{j} h^{i-k-j} \cap \Lambda_{i}(Z)
$$

and conversely:

$$
\Lambda_{k}(Z)=\sum_{j \geq 0}(-1)^{k+j}\left(\begin{array}{c}
k+j+1 \\
k+1
\end{array}\right) h^{j} \cap\left(\mathcal{M}_{k+j}(Z)+c_{1}\left(\left.L\right|_{Z}\right) \mathcal{M}_{k+j+1}(Z)\right) .
$$

The precise definition of the global Lê classes is given below. This uses a codimension $k+2$ linear subspace $L_{k+2}$ of $\mathbb{P}^{N}$ that defines the global Lê cycle $\Lambda_{k}\left(Z, L_{k+2}\right)$, which is an algebraic cycle that can be seen as the global counterpart of the local Lê cycles defined by Massey in [4]. The corresponding classes in Chow groups and homology groups are the global Lê classes in Theorem 1.1 and these are independent of the choice of a general linear space.

The justification of the moniker "global Lê cycle" is that for any given point of $Z$ there is a global Lê cycle $\Lambda_{k}\left(Z, L_{k+2}\right)$ whose restriction to a neighborhood $\mathcal{U}$ of the given point is the local Lê cycle, of the hypersurface $Z \cap \mathcal{U}$ at that point, defined by Massey in [4].

We notice too that the corollary following Theorem 1 remains unchanged, and so do, essentially, Theorems 2 and 4.7.

The concept of global Lê cycles of $Z$ that we gave in the original article was motivated by the Gaffney-Gassler interpretation in [2] of the local Lê cycles of holomorphic function-germs introduced by David Massey in [4]. These were defined as the Segre classes of $Z_{\text {sing }}$ in $M$, and we wrongly proved a theorem relating these to the Milnor classes of $Z$. In fact, a correct relation between Milnor and Segre classes had been proved by Aluffi in [1] at a higher level of generality. Our proof of Theorem 1 used a characterization of the global Lê cycles as Massey (or MacPherson) cycles, and that was the interpretation 
of the global Lê cycles that we actually used to prove our theorem. The first error was precisely the identification of these Segre classes with the Massey, or MacPherson, cycles (Eq. (14) in Proposition 4.6). Thence we must define the global Lê cycles differently, as indicated below.

We are grateful to Paolo Aluffi for pointing out to us that our main theorem in the original article could not be true as stated because it led to contradictions. We also thank him for allowing us to use his software for computing MacPherson and Segre classes of singular varieties.

Let $X$ be a $d$-dimensional subvariety of $\mathbb{P}^{N}$. Its $k$-th polar variety is (Definition 4.2):

$$
\mathbb{P}_{k}\left(X, L_{k+2}\right)=\overline{\left\{x \in X_{\text {reg }} \mid \operatorname{dim}\left(T_{x} X_{\text {reg }} \cap L_{k+2}\right) \geq d-k-1\right\}},
$$

where $L_{k+2}$ is a plane of codimension $k+2$ in $\mathbb{P}^{N}$ and $T_{x} X_{\text {reg }}$ is the projective tangent space of $X$ at a regular point $x$. The classes in Chow groups and homology represented by these polar varieties do not depend on the choice of the linear space provided this is sufficiently general (see [6, Proposition 1.2]). We denote these classes by $\left[\mathbb{P}_{k}(X)\right]$.

For any given constructible function $\beta$ on $X \subseteq \mathbb{P}^{N}$ with respect to a Whitney stratification $\mathcal{S}=\left\{S_{\alpha}\right\}$ of $X$, we define (following Schürmann and Tibăr in [7]) the MacPherson cycles as in Eq. (12):

$$
M P_{k}^{\mathbb{P}}\left(\beta, L_{k+2}\right):=\sum_{\alpha}(-1)^{d_{\alpha}} \eta\left(S_{\alpha}, \beta\right) \mathbb{P}_{k}\left(\bar{S}_{\alpha}, L_{k+2}\right),
$$

where $d_{\alpha}$ denotes the dimension of $S_{\alpha}$ and $\eta\left(S_{\alpha}, \beta\right)$ is the usual Normal Morse index (as in Definition 2.1).

Remark 1.2 On any compact algebraic variety $X$, Lê and Teissier in [3, Equation (6.1.5.3)] define a cycle $\Phi=\sum_{l} \sum_{j_{l} \in J_{l}} n_{l, j_{l}} F_{l, j_{l}}$, called also MacPherson cycle, such that $c^{M a}(\Phi)=c^{S M}(X)$. This cycle does not coincide with the (total) MacPherson cycle defined in this work as

$$
M P^{\mathbb{P}}\left(X, L_{\bullet}\right):=\sum_{k} \sum_{\alpha}(-1)^{d_{\alpha}} \eta\left(S_{\alpha}, 1_{X}\right) \mathbb{P}_{k}\left(\bar{S}_{\alpha}, L_{k+2}\right),
$$

where $\left\{S_{\alpha}\right\}$ is a Whitney stratification of $X$ and $L_{\bullet}: L_{N} \subseteq L_{N-1} \subseteq \cdots \subseteq$ $L_{0}=\mathbb{P}^{N}$ is a flag of linear subspaces $L_{j}$ of $\mathbb{P}^{N}$ of codimension $j$, because

$$
c^{M a}\left(M P^{\mathbb{P}}(X)\right) \neq c^{S M}(X)=\sum_{\alpha}(-1)^{d_{\alpha}} \eta\left(S_{\alpha}, 1_{X}\right) c^{M a}\left(\bar{S}_{\alpha}\right) .
$$


Definition 1.3 Consider the context of Theorem 1.1, with $Z$ endowed with a given Whitney stratification. The $k$ th global Lê cycle of $Z$ with respect to a general linear subspace $L_{k+2}$ (of codimension $k+2$ ) of $\mathbb{P}^{N}$ is:

$$
\Lambda_{k}\left(Z, L_{k+2}\right)=(-1)^{n} M P_{k}^{\mathbb{P}}\left(\omega, L_{k+2}\right),
$$

where $\omega(x)=\chi\left(F_{f, x}\right)-1$ and $f$, with $F_{f, x}$ a local Milnor fiber of $f$ at $x$ of the function $f$ corresponding to $s$ in some local trivialization of $L$ around $x$. The classes associated to these cycles will be denoted by $\Lambda_{k}(Z)$.

Notice that the Euler characteristic of the corresponding local Milnor fiber $F_{f, x}$ does not depend on the chosen trivialization of $L$. Moreover the function $\omega$ is constructible with respect to any given Whitney stratification of $Z$. Finally, the corresponding Lê cycle of $Z$ for a general linear subspace does not depend on the Whitney stratification, because the cycle $\sum_{\alpha} \eta\left(S_{\alpha}, \beta\right)\left[\mathbb{P}\left(T_{\bar{S}_{\alpha}}^{*} M\right)\right]$ does not depend on the choice of the stratification (see [7, Lemma 2.4]). Since $\mathbb{P}_{k}\left(\bar{S}_{\alpha}, L_{k+2}\right)=\pi_{*}\left(\mathbb{P}\left(T_{\bar{S}_{\alpha}^{*}}^{*} M\right) \cap\left(M \times L_{k+2}\right)\right)$, where $\pi: \mathbb{P}\left(T_{\bar{S}_{\alpha}^{*}}^{*} M\right) \rightarrow M$ is the projective conormal map, we have that the cycle

$$
M P_{k}^{\mathbb{P}}\left(\beta, L_{k+2}\right):=\sum_{\alpha}(-1)^{d_{\alpha}} \eta\left(S_{\alpha}, \beta\right) \mathbb{P}_{k}\left(\bar{S}_{\alpha}, L_{k+2}\right),
$$

does not depend on the stratification.

The following result expresses each Schwartz-MacPherson class through classes associated to the MacPherson cycles. The statement and the proof are as in Theorem 4.7.

Theorem 1.4 Let $X \subset \mathbb{P}^{N}$ be a subvariety endowed with a Whitney stratification with connected strata $S_{\alpha}$. If $\beta: X \rightarrow \mathbb{Z}$ is a constructible function with respect to this stratification and $\mathcal{L}:=\mathcal{O}_{\mathbb{P}^{N}}(1)$, then

$$
\begin{aligned}
c_{k}^{S M}(\beta) & =\sum_{\alpha} \eta\left(S_{\alpha}, \beta\right) \sum_{i=k}^{d_{\alpha}}(-1)^{d_{\alpha}-i}\left(\begin{array}{c}
i+1 \\
k+1
\end{array}\right) c_{1}\left(\left.\mathcal{L}\right|_{X}\right)^{i-k} \cap\left[\mathbb{P}_{i}\left(\bar{S}_{\alpha}\right)\right] \\
& =\sum_{i \geq k}(-1)^{i}\left(\begin{array}{c}
i+1 \\
k+1
\end{array}\right) c_{1}\left(\left.\mathcal{L}\right|_{X}\right)^{i-k} \cap M P_{i}^{\mathbb{P}}(\beta) .
\end{aligned}
$$

Recall that, for every $F^{\bullet}$ in $\mathcal{D}_{c}^{b}(X)$, the derived category of bounded, constructible complexes of sheaves of $\mathbb{C}$-vector spaces on $X$, the Massey cycles of $F^{\bullet}$ are (Definition 4.4):

$$
\Lambda_{k}^{\mathbb{P}}\left(F^{\bullet}, L_{k+2}\right)=\sum_{\alpha} m_{\alpha}\left(\psi_{\alpha}\right)_{*} \mathbb{P}_{k}\left(\bar{S}_{\alpha}, L_{k+2}\right),
$$


where $\psi_{\alpha}: \bar{S}_{\alpha} \hookrightarrow X$ is the inclusion and $m_{\alpha}=(-1)^{d-d_{\alpha}-1} \chi\left(\phi_{g_{\left.\right|_{N}}} F_{\left.\right|_{N}}^{\bullet}\right)_{p} ; g$ is a non-degenerate covector at $p \in S_{\alpha}$ with respect to the fixed stratification $\mathcal{S}=\left\{S_{\alpha}\right\}, N$ is a germ of a closed complex submanifold which is transversal to $S_{\alpha}$ with $N \cap S_{\alpha}=\{p\}$ and $\phi_{g_{\left.\right|_{N}}} F_{\left.\right|_{N}}^{\bullet}$ is the sheaf of vanishing cycles of $F_{||_{N}}^{\bullet}$ with respect to $g_{\left.\right|_{N}}$. When $\beta(p)=\chi\left(F^{\bullet}\right)_{p}$ for $F^{\bullet} \in \mathcal{D}_{\mathcal{S}}^{b}(X)$ we denote $\Lambda_{k}^{\mathbb{P}}\left(F^{\bullet}\right)$ also by $\Lambda_{k}^{\mathbb{P}}(\beta)$. In this case, by Proposition 4.6 (13) we have

$$
\Lambda_{k}^{\mathbb{P}}\left(F^{\bullet}, L_{k+2}\right)=(-1)^{\operatorname{dim} X} M P_{k}^{\mathbb{P}}\left(\beta, L_{k+2}\right) .
$$

Remark 1.5 This equality follows from Remark 4.5 because two projective cycles coincide if and only if their pullback to every chart in an affine open covering coincide. The non trivial part of this statement is not true for cycle classes in Chow groups, and that was the argument we used wrongly in the original article to state Eq. (14). Notice that the second equation of Theorem 1.1 relates the global Lê cycles with the Milnor classes of $Z$, hence by using [1, Theorem I.1] we get a description of the global Lê cycles in terms of Segre classes of the singular locus of $Z$ in $M$.

The classes associated to the MacPherson and Massey cycles will be denoted by $M P_{k}^{\mathbb{P}}(\beta)$ and $\Lambda_{k}^{\mathbb{P}}\left(F^{\bullet}\right)$ respectively. Thus,

$$
\begin{aligned}
M P_{k}^{\mathbb{P}}(\beta) & =\sum_{\alpha}(-1)^{d_{\alpha}} \eta\left(S_{\alpha}, \beta\right)\left[\mathbb{P}_{k}\left(\bar{S}_{\alpha}\right)\right] \text { and } \\
\Lambda_{k}^{\mathbb{P}}\left(F^{\bullet}\right) & =\sum_{\alpha} m_{\alpha}\left(\psi_{\alpha}\right)_{*}\left[\mathbb{P}_{k}\left(\bar{S}_{\alpha}\right)\right] .
\end{aligned}
$$

By Corollary 2.4 and (2) we have that the global Lê cycles are the compactification of the global affine MacPherson cycles introduced by Schürmann and Tibăr in [7].

With this definition of global Lê cycles, Lemma 6.1 becomes the lemma below, with essentially the same proof. This leads to the first equation in Theorem 1.1.

Lemma 1.6 (Main Lemma) Let $M$ and $Z$ be as in Theorem 1.1. Then

$$
\mathcal{M}_{k}(Z)=\sum_{j \geq 0} \sum_{i \geq k+j}(-1)^{i+j}\left(\begin{array}{c}
i+1 \\
k+j+1
\end{array}\right) c_{1}\left(\left.L\right|_{Z}\right)^{j} h^{i-k-j} \cap \Lambda_{i}(Z) .
$$

We get: 


\section{Corollary 1.7}

$$
\begin{aligned}
& \Lambda_{k}(Z) \\
& =\sum_{j \geq 0}(-1)^{k+j}\left(\begin{array}{c}
k+j+1 \\
k+1
\end{array}\right) h^{j} \cap\left(\mathcal{M}_{k+j}(Z)+c_{1}\left(\left.L\right|_{Z}\right) \mathcal{M}_{k+j+1}(Z)\right) .
\end{aligned}
$$

Proof Notice that, by Lemma 1.6,

$$
\mathcal{M}_{k+l}+c_{1}\left(\left.L\right|_{Z}\right) \cap \mathcal{M}_{k+l+1}=\sum_{i \geq k+l}(-1)^{i}\left(\begin{array}{c}
i+1 \\
k+l+1
\end{array}\right) h^{i-k-l} \cap \Lambda_{i}(Z)
$$

Hence, it is enough to prove that

$$
\sum_{l \geq 0} \sum_{i \geq k+l}(-1)^{k+l+i}\left(\begin{array}{c}
k+l+1 \\
k+1
\end{array}\right)\left(\begin{array}{c}
i+1 \\
k+l+1
\end{array}\right) h^{i-k} \cap \Lambda_{i}(Z)=\Lambda_{k}(Z)
$$

which follows by simple combinatorial manipulations, as in the proof of Lemma 6.5.

This completes the proof of Theorem 1.1.

Example 1.8 Let $Z$ be the hypersurface of $\mathbb{P}^{4}$ defined by the homogeneous polynomial $H\left(x_{0}, \ldots, x_{4}\right)=x_{3} x_{4}$. Consider the following Whitney stratification of $Z: S_{1}=V\left(x_{3}\right) \backslash V\left(x_{3}, x_{4}\right) ; S_{2}=V\left(x_{4}\right) \backslash V\left(x_{3}, x_{4}\right) ; \quad S_{3}=$ $V\left(x_{3}, x_{4}\right) \backslash V\left(x_{2}, x_{3}, x_{4}\right) ; \quad S_{4}=V\left(x_{2}, x_{3}, x_{4}\right) \backslash V\left(x_{1}, x_{2}, x_{3}, x_{4}\right) ; S_{5}=$ $V\left(x_{1}, x_{2}, x_{3}, x_{4}\right)$. Notice that the polar varieties of the strata are: $\mathbb{P}_{k}\left(\overline{S_{j}}\right)=\emptyset$ if $k \neq \operatorname{dim}\left(\overline{S_{j}}\right)$ and $\mathbb{P}_{k}\left(\overline{S_{j}}\right)=\overline{S_{j}}$ if $k=\operatorname{dim}\left(\overline{S_{j}}\right)$. Therefore,

$$
M P_{k}^{\mathbb{P}}(\omega)=\eta\left(S_{5-k}, \omega\right)\left[\mathbb{P}^{k}\right], \quad k=0,1,2,
$$

where $\omega(x)=\chi\left(F_{f, x}\right)-1$ with $f$ being the restriction of $H$ to a neighborhood of $x$ and $F_{f, x}$ being the Milnor fiber of $f$ at $x$. It remains to compute the normal Morse index of the strata. For example, for $S_{3}$ the complex link is given by $l_{S_{3}}=Z \cap N \cap B_{\delta}(p) \cap\{g=t\}$ for $0<|t| \ll \delta \ll 1$, where $p=(0: 0: 1: 0: 0), N=V\left(x_{0}, x_{1}\right)$ and $g=x_{3}+x_{4}$. Thus, $l_{S_{3}}=\left\{p_{1}, p_{2}\right\}$, where $p_{1}=(0: 0: 1: 0: t)$ and $p_{2}=(0: 0: 1: t: 0)$. Hence

$$
\eta\left(S_{3}, \omega\right)=\omega(p)-\omega\left(p_{1}\right) \chi\left(p_{1}\right)-\omega\left(p_{2}\right) \chi\left(p_{2}\right)=-1-0-0=-1 .
$$

Analogously, we obtain that $\eta\left(S_{4}, \omega\right)=\eta\left(S_{5}, \omega\right)=0$. Therefore $M P_{2}^{\mathbb{P}}(\omega)=$ $-\left[\mathrm{P}^{2}\right]$ and the other are all zero.

We may compute the Milnor classes of $Z$ using the formula given in Lemma 1.6. We get that $\mathcal{M}_{k}(Z)=\left[\mathbb{P}^{k}\right]$ for $k=0,1,2$. 
In the original article we discussed too the relation between the global Lê cycles and the Aluffi classes of the singular set of $Z$. These are $\alpha_{Z_{\text {sing }}}=$ $c\left(L_{\mid Z}\right) \cap \mathcal{M}(Z)$ (cf. Eq. (21)). From Eq. (3) above we get that the correct statement of Corollary 6.7 is.

Corollary 1.9 $\left(\alpha_{Z_{\text {sing }}}\right)_{k}=\sum_{l \geq 0}(-1)^{k+l}\left(\begin{array}{c}k+l+1 \\ k+1\end{array}\right) h^{l} \cap \Lambda_{k+l}(Z)$.

\section{References}

1. Aluffi, P.: Chern classes for singular hypersurfaces. Trans. Am. Math. Soc. 351, 3989-4026 (1999)

2. Gaffney, T., Gassler, R.: Segre numbers and hypersurface singularities. J. Algebr. Geom. 08, 695-736 (1999)

3. Lê, D.T., Teissier, B.: Variétés polaires locales et classes de Chern des variétés singulieres. Ann. Math. 114(2), 457-491 (1981)

4. Massey, D.B.: Lê Cycles and Hypersurface Singularities. Lecture Notes in Mathematics, vol. 1615. Springer, Berlin (1995)

5. Piene, R.: Cycles polaires et classes de Chern pour les variétés projectives singulieres. Travaux en Cours 37, 7-34 (1988)

6. Piene, R.: Polar classes of singular varieties. Ann. Sci. École Norm. Sup. 4 11(2), 247-276 (1978)

7. Schürmann, J., Tibăr, M.: Index formula for MacPherson cycles of affine algebraic varieties. Tohoku Math. J. 62, 29-44 (2010) 